\title{
Brain cholesterol in normal and pathological aging
}

\author{
Tim VANMIERLO ${ }^{1,2}$ \\ Dieter LÜTJOHANN ${ }^{1}$ \\ Monique MULDER ${ }^{3}$ \\ ${ }^{1}$ Institute of Clinical Chemistry and \\ Clinical Pharmacology, University of \\ Bonn, Sigmund-Freud-Str. 25, D-53127 \\ Bonn, Germany \\ ${ }^{2}$ Department of Neuroscience, \\ Maastricht University, Universiteitssingel \\ 50, 6229 ER, Maastricht \\ ${ }^{3}$ Department of Internal Medicine, \\ Division of Cardiovascular Diseases, \\ Erasmus MC, 's-Gravendijkwal 230, \\ 3015 CE Rotterdam, The Netherlands \\ $<$ m.t.mulder@erasmusmc.nl >
}

\begin{abstract}
Aberrations in cerebral cholesterol homeostasis can lead to severe neurological diseases. Recent findings strengthen the link between brain cholesterol metabolism and factors involved in synaptic plasticity, a process essential for learning and memory functions, as well as regeneration, which are affected in Alzheimer's Disease (AD). Cholesterol homeostasis within the brain is independent of that in the rest of the body and needs to be strictly regulated for optimal brain functioning. In contrast with what was initially assumed brain cholesterol homeostasis can be modulated by extra-cerebral factors. We have found that enhancement of the cholesterol-turnover in the brain by administration of the synthetic activator of liver $x$ receptos (LXRs), T0901317, leads to restoration of memory functions in an AD mouse-model. Memory in C57BI6NCrl mice was not further improved by the same treatment. Moreover, it was found that in contrast with cholesterol, the structurally very similar dietary derived plant sterols can enter the brain. Plant sterols may be natural activators of LXRs. Evidence is provided suggesting that brassicasterol may be a novel additional biomarker in cerebrospinal fluid of AD patients. Insight into the regulation of cerebral cholesterol homeostasis will provide possibilities to modulate the key steps involved and may lead to the development of therapies for the prevention as well as treatment of neurodegenerative diseases such as $A D$.
\end{abstract}

Key words: Alzheimer's Disease, cholesterol, liver x receptors, plant sterols, memory

\begin{abstract}
Aberrations in cerebral cholesterol homeostasis can lead to severe neurological diseases and have recently been linked to Alzheimer's Disease (AD) (Maxfield and Tabas, 2005; Tint et al., 1995; Marx, 2001; Puglielli et al., 2004). $A D$ is a slowly progressing neurodegenerative disease that is neuropathologically characterized by senile plaques, with amyloid- $\beta$ as a key protein, neurofibrillary tangles, loss of synapses, and often by vascular dysfunction and inflammatory processes. Recent findings strengthen the link between brain cholesterol metabolism and factors involved in synaptic plasticity, a process essential for learning and memory functions. A number of the known risk factors for $A D$ are related to cholesterol metabolism. APOE4, one of the three common isoforms of APOE, is the strongest known genetic risk factor for
\end{abstract}

AD (Corder et al., 1993). Apolipoprotein $\mathrm{E}$ (apoE) is best known for its role in cholesterol trafficking in the periphery and it is thought to exert a similar function within the central nervous system (Pitas et al., 1987). Additionally, hypercholesterolemia at middle age and a high fat intake have been associated with an increased risk of $A D$, and use of statins, cholesterol synthesis lowering agents, have been associated with a reduced risk (Haag et al., 2008; Wolozin, 2004) Although, the latter remains controversial and it is unlikely that statins exert their effects via an inhibition of the cholesterol synthesis rate within the brain. Moreover, the lipid composition of membranes, including the cholesterol level, has been reported to affect the splicing of amyloid from its trans-membrane precursor protein (Puglielli et al., 2001; Frears et al., 1999; Simons et al., 1998). Interestinly, the cholesterol synthesis rate within the brain decreases with age and has been suggested to be associated with an increased decline of memory functions (Thelen et al., 2006).

Many proteins involved in peripheral cholesterol metabolism are also present in the brain. Yet, brain cholesterol metabolism is very different from that in the remainder of the body. The brain makes up about $2 \%$ of the total body weight, but contains almost $25 \%$ of all free cholesterol. Lipoproteins present in the circulation are prevented from entering the brain by the blood-brain barrier and thus all cholesterol is synthesized endogenously predominantly by astrocytes (Dietschy and Turley, 2001; Legleiter et al., 2004; Xu et al., 2006). There is a daily synthesis of at

To cite this article: Vanmierlo T, Lütjohann D, Mulder M. Brain cholesterol in normal and pathological aging. OCL 2011; 18(4): 214-7. doi : 10.1684/ocl.2011.0398 
least $6 \mathrm{mg}$ of cholesterol in the brain. Because cholesterol cannot be degraded and high amounts of free cholesterol are toxic to cells, neurons in particular, the excess is secreted from the brain. About $60 \%$ is secreted in the form of brain specific 24(S)-hydroxycholesterol, a more polar metabolite of cholesterol (Lund et al., 1999; Babiker et al., 1997; Bjorkhem et al., 1997, Bjorkhem et al., 1998; Lutjohann et al., 1996). The neuron-specific enzyme cholesterol 24S-hydroxylase, CYP46A1, is responsible for the conversion. In addition apoE-containing high density lipoprotein-like particles may be involved in the secretion of the remaining $40 \%$ of cholesterol from the brain either directly into the circulation or via the cerebrospinal fluid (CSF) (Rebeck, 2004; Shafaati et al., 2007).

\section{Trafficking of cholesterol between astrocytes and neurons}

There is evidence indicating that neurons largely shut down their cholesterol synthesis after birth and rely on astrocytes for their cholesterol supply, because they would need their energy for maintenance of ion-gradients across membranes for electrical and chemical signaling (Pfrieger, 2003; Posse De Chaves et al., 2000). Neurons require cholesterol for a number of different functions including vesicle transport, neurotransmitter release and as a precursor for neurosteroids. Moreover, they need cholesterol for the generation of new membranes for example for the formation of new synapses during a process called synaptic plasticity; the reorganization of synaptic contacts between neuronal axons and dendrites or somata of other neurons which occurs during learning (Pfrieger, 2003). Cholesterol is being secreted by astrocytes in the form of high density lipoprotein (HDL)-like particles containing apoE as a major protein (Legleiter and LaDu, 1998; Gong 2002). The particles secreted by astrocytes differ slightly from those in plasma with respect to their size, shape and aggregation properties. We have recently shown that 24(S)-hydroxycholesterol that is being secreted by neurons is involved in regulating this process, by stimulating the secretion of apoE-containing HDL-like particles from astro- cytes (Abildayeva et al., 2006). 24(S)hydroxycholesterol activates so-called liver $X$ receptors (LXRs), which are master regulators of cholesterol homeostasis. LXRs belong to the nuclear hormone receptor superfamily, of which the two forms, LXR $\alpha$ and $L X R \beta$, are present in the brain (Whitney et al., 2002; Eckert et al., 2007). This results in induction of apoE production and secretion, and in an enhanced cholesterol secretion. Because the expression of the ATP-binding cassette transporters $A 1$ and $\mathrm{G} 1$ was upregulated, these may be involved in controlling the secretion.

\section{Cerebral cholesterol homeostasis as a therapeutical target in Alzheimer's Disease?}

The process of cholesterol supply from astrocytes to neurons may be compromised in AD. A number of genes encoding proteins involved in this process, have been associated with $A D$, such as APOE, ABCA1, CYP46A1. Increased plasma and CSF levels of 24 (S)-hydroxycholesterol have been found in early stages of $A D$, while reduced levels were observed in later stages, possibly due to the loss of CYP46A1-expressing neurons, which are the metabolically active ones (Heverin et al., 2004; Papassotiropoulos et al., 2002; Lutjohann et al., 2000). Moreover, the levels of HDL-like particles were found to be strongly reduced in post mortem CSF of AD patients (Papassotiropoulos et al., 2002; Mulder et al., 1998).

As mentioned, a strict regulation of brain cholesterol homeostasis is required for optimal brain functioning. The autonomous regulation of brain cholesterol homeostasis is demonstrated by the observation that apoE-deficient mice that are being fed a high fat diet and display about 20-fold increased plasma cholesterol levels, show no alterations in their brain sterol profile in comparison with wild-type chow-fed mice, with the exception of a slight increase in 27hydroxycholesterol levels (Jansen et al., 2009). However, although tightly regulated, there are extracerebral factors that do modulate cerebral cholesterol homeostasis.

Besides evidence that an aberrant cholesterol metabolism in the brain may contribute to the progression of $A D$, we found alterations in the sterol profile in the brain of AD-mice expressing a mutant form of APP and of PS1 (Vanmierlo et al., 2010). This indicates that alterations in brain cholesterol metabolism can also be induced by the expression of APP- and/or PS1 mutations in mice, and is supportive of a close link between cholesterol and AD. Stimulation of the LXR-pathway, that plays a key role in the regulation of cholesterol metabolism in the body and the brain results in an enhanced cholesterol turnover in the body and also in the brain (Repa et al., 2007; Plosch et al., 2002). LXR-activation has been reported to reduce the deposition of amyloid$\beta$, the key protein of senile plaques in AD-brains (Koldamova et al., 2005; Sun et al., 2003). This may involve several mechanisms such as reducing the soluble levels, enhancing its secretion from the brain, and stimulation of $A \beta$ degradation by microglia (Bell et al., 2007, Mulder and Terwel, 1998). We questioned if enhancement of the cholesterol turnover in the brain, by administration of the synthetic LXR agonist T0901317, would restore memory functions in aged AD-mice. Herefore, we fed 21 week-old AD-mice a diet containing T0901317 (0.015\% drug in food, w/w) for a period of 10 weeks (Vanmierlo et al., 2009). This resulted in an enhanced cholesterol turnover in the brain as indicated by increased levels of the cholesterol precursors, desmosterol, lathosterol and lanosterol in the cortex, hippocampus and cerebellum, and in upregulation of a number of LXR-target genes involved in cellular cholesterol secretion including Abca1, Abcg1 Apoe and Srebp1c. T0901317 administration resulted in an improved performance of the mice in the Object Recognition Task, showing that activation of the LXR-pathway could restore the declined memory in the AD-mice. There was no detectable effect of T0901317 on the amyloid load in the brain in any of the brain regions examined, e.g. cortex, hippocampus and prelimbic area. T0901317 did not further improve memory in $\mathrm{C} 57 \mathrm{Bl} 6$ wildtype mice. Conclusively, T0901317 restored, at least in part, memory in aged AD-mice, independently of an effect on amyloid deposition. LXR agonists were originally developed as drugs that lower plasma cholesterol by enhancing its secretion from the body. However 
because of serious side effects of triglyceride accumulation in the liver, novel more selective LXR agonists are being developed (Giannarelli et al., 2011).

\section{Plant sterols derived from the diet can enter the brain}

Plant sterols have been suggested to be natural activators of the LXR-pathway and therefore, may enhance the cholesterol turnover (Plat et al., 2005; Yang, 2006). The most common plant sterols, such as sitosterol and campesterol, are structurally very similar to cholesterol and can only be derived from the diet (Pollak and Kritchevsky, 1981; Salen et al., 1970). They are applied as functional food in order to lower plasma cholesterol levels as a strategy to prevent cardiovascular diseases (Thompson and Grundy, 2005; CalpeBerdiel et al., 2009). We found that plant sterols, in contrast with cholesterol, can cross the blood-brain barrier and enter the brain in Abc5-knockout mice (Jansen et al., 2006; Fricke et al., 2007). The Abcg5 transporter is a predominantly intestinal receptor that forms heterodimers with Abcg8 in order to selectively resecrete plant sterols in the intestine (Yu et al., 2002). In case of deficiency for one of these transporters plant sterols are being retained in the intestine and released into the circulation resulting in increased plasma levels. Abcg5-knockout mice display 35-70fold increased plant sterol levels in plasma and 5-12-fold increased levels in their brains, in hippocampus, cortex and cerebellum. Campesterol preferentially accumulates in the brain in hippocampus and cortex, and to the highest extend in the cerebellum. Although, plant sterol levels were increased in all brain regions, a small but significant effect on brain sterols was observed in restricted brain regions; levels of the cholesterol precursor, lanosterol, were increased in the cortex and to a lesser extend in hippocampus, and levels of the cholesterol precursor desmosterol and of the cholesterol metabolite 24(S)hydroxycholesterol were reduced in the hippocampus, a brain region involved in learning- and memory functions. The latter was in contrast with our expectations that plant sterols may enhance the cholesterol turnover by activating the LXR-pathway. The reduced desmos- terol and 24(S)-hydroxycholesterol levels suggest a reduced cholesterol turnover. However, subjecting Abcg5knockout mice and their wild-type littermates to behavioral tasks, revealed no differences in memory functions or in anxiety and mood-related behavior. An exception was the swimming speed, which was slightly higher in the Abcg5knockout mice in comparison with their wild-type littermates (Vanmierlo et al., 2011a). Therefore, it can be concluded that accumulation of plant sterols in the Abcg5-knockout mice does not lead to an overt behavioral phenotype of impairments in memory functions or in mood and anxiety related behavior. The elevated plant sterol levels in the brain were accompagnied by an increased expression of Abca1 and Abcg1 in particular in the hippocampus, but the expression of other LXR-target genes was unaffected, suggesting the mechanisms involved in upreglating Abca1 and Abcg1 are LXR-independent. So far, no evidence was obtained for a major effect of plant sterols as LXR activators within the brain.

Abcg5 and Abcg8, the transporters for plant sterols, are not detectable in the brain. We obtained evidence indicating that the plant sterols that have entered the brain are not being resecreted.

\section{Brassicasterol; a novel biomarker for Alzheimer's Disease?}

Very recently, evidence was obtained indicating that brassicasterol, a plant sterol less common than sito- and campesterol, may be an additional biomarker for AD (Vanmierlo et al., 2011b). In the early stages of the disease the functions of the blood-brain barrier and of the choroid plexus are impaired. We hypothesized that as a consequence, plant sterol concentrations may be altered in AD CSF. Indeed plant sterol concentrations turned out to be reduced in CSF of AD patients in comparison with controls. Both sitosterol and brassicasterol were significantly reduced, but only the difference for brassicasterol remained signifant after correction for cholesterol. Brassicasterol improved the predictive value when added to the biomarkers pTau and A $\beta 42$. Thus brassicasterol might be a relevant additional biomarker for AD.
Conclusively, sterol metabolism in the brain may be a promising therapeutical target in the prevention and/or retardation of $\mathrm{AD}$ and it therefore may be worthwhile to continue investigating LXR agonists that are being developed without serious side effects. Moreover, it is important to further investigate the consequences of elevated brain plant sterol levels, and it remains to be established how elevated cerebral plant sterol levels affect neuropathogenesis such as for example during the progression of $A D$. As decribed for LXR activation by T0901317 that did restore impaired memory functions in AD-mice but did not further improve these in wild-type mice, plant sterols may affect cognitive functions or neuropathogenesis in AD-mice.

\section{REFERENCES}

Abildayeva K, et al. 24(S)-hydroxycholesterol participates in a liver $\mathrm{X}$ receptor-controlled pathway in astrocytes that regulates apolipoprotein E-mediated cholesterol efflux. J Biol Chem 2006; 281: 12799-808.

Babiker A, et al. Elimination of cholesterol in macrophages and endothelial cells by the sterol 27-hydroxylase mechanism. Comparison with high density lipoprotein-mediated reverse cholesterol transport. J Biol Chem 1997; 272: 26253-61.

Bell RD, et al. Transport pathways for clearance of human Alzheimer's amyloid beta-peptide and apolipoproteins $\mathrm{E}$ and $\mathrm{J}$ in the mouse central nervous system. / Cereb Blood Flow Metab 2007; 27: 909-18.

Bjorkhem I, et al. Importance of a novel oxidative mechanism for elimination of brain cholesterol. Turnover of cholesterol and 24 (S)-hydroxycholesterol in rat brain as measured with 1802 techniques in vivo and in vitro. I Biol Chem 1997; 272: 30178-84.

Bjorkhem I, et al. Cholesterol homeostasis in human brain: turnover of 24S-hydroxycholesterol and evidence for a cerebral origin of most of this oxysterol in the circulation. J Lipid Res 1998; 39: 1594-600.

Calpe-Berdiel L, Escola-Gil JC, Blanco-Vaca F. New insights into the molecular actions of plant sterols and stanols in cholesterol metabolism. Atherosclerosis 2009; 203: 18-31.

Corder $\mathrm{EH}$, et al. Gene dose of apolipoprotein E type 4 allele and the risk of Alzheimer's disease in late onset families. Science 1993; 261: 921-3.

Dietschy JM, Turley SD. Cholesterol metabolism in the brain. Curr Opin Lipidol 2001; 12: 105-12. 
Eckert GP, et al. Regulation of central nervous system cholesterol homeostasis by the liver $\mathrm{X}$ receptor agonist TO-901317. Neurosci Lett 2007; 423: 47-52.

Frears ER, et al. The role of cholesterol in the biosynthesis of beta-amyloid. Neuroreport 1999; 10: 1699-705.

Fricke $C B$, et al. Increased plant sterol and stanol levels in brain of Watanabe rabbits fed rapeseed oil derived plant sterol or stanol esters. Br J Nutr 2007; 98: 890-9.

Giannarelli $C$, et al. Synergistic effect of liver $X$ receptor activation and simvastatin on plaque regression and stabilization: an magnetic resonance imaging study in a model of advanced atherosclerosis. Eur Heart J 2011.

Gong JS, et al. Apolipoprotein E (ApoE) isoform-dependent lipid release from astrocytes prepared from human ApoE3 and ApoE4 knock-in mice. I Biol Chem 2002; 277: 29919-26.

Haag MD, et al. Cyclooxygenase selectivity of nonsteroidal anti-inflammatory drugs and risk of stroke. Arch Intern Med 2008; 168: 1219-24.

Heverin $M$, et al. Changes in the levels of cerebral and extracerebral sterols in the brain of patients with Alzheimer's disease. / Lipid Res 2004; 45: 186-93.

Jansen PJ, et al. Dietary plant sterols accumulate in the brain. Biochim Biophys Acta 2006; 1761: 445-53.

Jansen PJ, et al. Absence of ApoE upregulates murine brain ApoD and ABCA1 levels but does not affect brain sterol levels while human ApoE3 and human ApoE4 upregulate brain cholesterol precursor levels. J Alzheimers Dis 2009; 18: 319-29.

Koldamova RP, et al. The liver $\mathrm{X}$ receptor ligand T0901317 decreases amyloid beta production in vitro and in a mouse model of Alzheimer's disease. / Biol Chem 2005; 280: 4079-88.

LaDu MJ, et al. Nascent astrocyte particles differ from lipoproteins in CSF. J Neurochem 1998; 70: 2070-81.

Legleiter J, et al. In situ AFM studies of astrocyte-secreted apolipoprotein E- and J-containing lipoproteins. I Colloid Interface Sci 2004; 278: 96-106.

Lund EG, Guileyardo JM, Russell DW. cDNA cloning of cholesterol 24-hydroxylase a mediator of cholesterol homeostasis in the brain. Proc Natl Acad Sci U S A 1999; 96: 7238-43.

Lutjohann D, et al. Cholesterol homeostasis in human brain: evidence for an age-dependent flux of 24S-hydroxycholesterol from the brain into the circulation. Proc Natl Acad Sci U S A 1996; 93: 9799-804.
Lutjohann D, et al. Plasma 24S-hydroxycholesterol (cerebrosterol) is increased in Alzheimer and vascular demented patients. J Lipid Res 2000; 41: 195-8.

Marx J. Alzheimer's disease. Bad for the heart bad for the mind? Science 2001; 294: 508-9.

Maxfield FR, Tabas I. Role of cholesterol and lipid organization in disease. Nature 2005; 438: 612-21.

Mulder M, et al. Reduced levels of cholesterol phospholipids and fatty acids in cerebrospinal fluid of Alzheimer disease patients are not related to apolipoprotein E4. Alzheimer Dis Assoc Disord 1998; 12: 198-203.

MulderM, TerwelD. Possible link between lipid metabolism and cerebral amyloid angiopathy in Alzheimer's disease: A role for high-density lipoproteins? Haemostasis 1998; 28: 174-94.

Papassotiropoulos A, et al. 24S-hydroxycholesterol in cerebrospinal fluid is elevated in early stages of dementia. J Psychiatr Res 2002; 36: $27-32$

Pfrieger FW. Outsourcing in the brain: do neurons depend on cholesterol delivery by astrocytes? Bioessays 2003; 25: 72-8.

Pitas RE, et al. Lipoproteins and their receptors in the central nervous system. Characterization of the lipoproteins in cerebrospinal fluid and identification of apolipoprotein $\mathrm{BE}(\mathrm{LDL})$ receptors in the brain. / Biol Chem 1987; 262: 14352-60.

Plat J, Nichols JA, Mensink RP. Plant sterols and stanols: effects on mixed micellar composition and LXR (target gene) activation. J Lipid Res 2005; 46: 2468-76.

Plosch T, et al. Increased hepatobiliary and fecal cholesterol excretion upon activation of the liver $\mathrm{X}$ receptor is independent of $\mathrm{ABCA} 1$. J Biol Chem 2002; 277: 33870-7.

Pollak OJ, Kritchevsky D. Sitosterol. Monogr Atheroscler 1981; 10: 1-219.

Posse De Chaves El, et al. Uptake of lipoproteins for axonal growth of sympathetic neurons. J Biol Chem 2000; 275: 19883-90.

Puglielli L, et al. Acyl-coenzyme A: cholesterol acyltransferase modulates the generation of the amyloid beta-peptide. Nat Cell Biol 2001; 3: 905-12.

Puglielli L, et al. Role of acyl-coenzyme a: cholesterol acyltransferase activity in the processing of the amyloid precursor protein. J Mol Neurosci 2004; 24: 93-6.

Rebeck GW. Cholesterol efflux as a critical component of Alzheimer's disease pathogenesis. J Mol Neurosci 2004; 23: 219-24.

Repa JJ, et al. Liver $\mathrm{X}$ receptor activation enhances cholesterol loss from the brain decreases neuroinflammation and increases survival of the NPC1 mouse. J Neurosci 2007; 27: $14470-80$.
Salen G, Ahrens Jr. EH, Grundy SM. Metabolism of beta-sitosterol in man. I Clin Invest 1970; 49: 952-67.

Shafaati M, et al. Levels of ApoE in cerebrospinal fluid are correlated with Tau and 24Shydroxycholesterol in patients with cognitive disorders. Neurosci Lett 2007; 425: 78-82.

Simons $\mathrm{M}$, et al. Cholesterol depletion inhibits the generation of beta-amyloid in hippocampal neurons. Proc Natl Acad Sci U S A 1998; 95: 6460-4.

Sun $Y$, et al. Expression of liver $X$ receptor target genes decreases cellular amyloid beta peptide secretion. / Biol Chem 2003; 278: 27688-94

Thelen KM, et al. Cholesterol synthesis rate in human hippocampus declines with aging. Neurosci Lett 2006; 403: 15-9.

Thompson GR, Grundy SM. History and development of plant sterol and stanol esters for cholesterol-lowering purposes. Am / Cardiol 2005; 96: 3D-9D.

Tint GS, et al. Correlation of severity and outcome with plasma sterol levels in variants of the Smith-Lemli-Opitz syndrome. J Pediatr 1995; 127: 82-7.

Vanmierlo $\mathrm{T}$, et al. Liver $\mathrm{X}$ receptor activation restores memory in aged $A D$ mice without reducing amyloid. Neurobiol Aging 2009.

Vanmierlo $\mathrm{T}$, et al. Alterations in brain cholesterol metabolism in the APPSLXP. S1mut mouse a model for Alzheimer's disease. / Alzheimers Dis 2010; 19: 117-27.

Vanmierlo T, et al. Cerebral Accumulation of Dietary Derivable Plant Sterols does not Interfere with Memory and Anxiety Related Behavior in Abcg5-/- Mice. Plant Foods Hum Nutr 2011a.

Vanmierlo $\mathrm{T}$, et al. The plant sterol brassicasterol as additional CSF biomarker in Alzheimer's disease. Acta Psychiatr Scand 2011 b.

Whitney KD, et al. Regulation of cholesterol homeostasis by the liver $\mathrm{X}$ receptors in the central nervous system. Mol Endocrinol 2002; 16: 1378-85.

Wolozin B. Cholesterol and the biology of Alzheimer's disease. Neuron 2004; 41: 7-10.

$\mathrm{Xu} \mathrm{Q}$, et al. Profile and regulation of apolipoprotein $E$ (ApoE) expression in the CNS in mice with targeting of green fluorescent protein gene to the ApoE locus. I Neurosci 2006; 26: 4985-94.

Yang $\mathrm{H}$. Nonvesicular sterol transport: two protein families and a sterol sensor? Trends Cell Biol 2006. 16: 427-32.

Yu L, et al. Disruption of Abcg5 and Abcg8 in mice reveals their crucial role in biliary cholesterol secretion. Proc Natl Acad Sci U S A 2002; 99: 16237-42. 